\title{
PNPRS OFFICERS AND MEMBERS 2018-2019
}

\section{Officers}

1. Executive Governor - Dr. Jove Jim S. Aguas (University of Santo Tomas, Manila)

2. Chairman - Dr. Juan Rafael G. Macaranas (De La Salle-College of Saint Benilde, Manila)

3. Vice Chairman - Dr. Danilo S. Alterado (Saint Louis University, Baguio)

4. Secretary - Mr. Jeffrey L. Bartilet (Polytechnic University of the Philippines, Manila)

5. Treasurer - Mr. Alvin O. Tan (University of Santo Tomas, Manila)

6. Auditor - Dr. Fleurdeliz R. Altez-Albela (University of Santo Tomas, Manila)

7. Public Relations Officer- Dr. Rodrigo D. Abenes (Philippine Normal University, Manila)

8. Legal Officer - Ms. Ma. Glovedi Joy L. Bigornia (Philippines Military Academy, Baguio)

9. Administrative Officer - Ms. Basilisa P. Macaranas

\section{Members}

10. Dr. Peter M. Collins (Institute for the History of Philosophy and Pedagogy, Maryland)

11. Dr. Noelle Leslie Dela Cruz (De La Salle University, Manila)

12. Dr. Leni dlR. Garcia (De La Salle University, Manila)

13. Dr. James M. Lawler (Retired, State University of New York, Buffalo, USA)

14. Dr. Napoleon M. Mabaquiao, Jr. (De La Salle University, Manila)

15. Dr. Olatunji Oyeshile (University of Ibadan, Nigeria)

16. Dr. Narcisa Paredes-Canilao (University of the Philippines, Baguio City)

17. Dr. Wilfried M.A. Vanhoutte (Saint Louis University, Baguio)

18. Mr. Emerson S. Abreu

19. Ms. Ninotchka Mumtaj Albano (De La Salle University - Manila)

20. Mr. Bryan B. Albia (San Beda University, Manila)

21. D. Wilhelm Julian Alfredo (Department of Agrarian Reform, Quezon City)

22. Dr. Emmanuel Ifeanyi Ani (University of Ghana, Legon)

23. Fr. Richie L. Armas, SMAHE (Holy Family Center of Studies Foundation, Sorsogon)

24. Mr. Conrado Baday B. Asejo (Saint Louis University, Baguio City/DepEd)

25. Dr. Julmar O. Asilo (Manuel Enverga University Foundation, Lucena City)

26. Mr. Victoriano Barliso, Jr. (Philippine Normal University, Manila)

27. Dr. Ufuk Özen Baykent (Uludag University, Turkey)

28. Ms. Roxanne T. Bongco (Bataan Peninsula State University, Bataan)

29. Mr. Elmer Palencia Brabante (Polytechnic University of the Philippines, Manila)

30. Mr. Darren Anthony O. Cabildo (Freelancer)

31. Fr. Shierwin Agagen Cabunilas (Diocese of Baguio/Institute of Philosophy, Belgium)

32. Dr. Mark Joseph Calano (Ateneo de Manila University, Quezon City)

33. Mr. Jonathan James O. Cañete (Pasig Catholic College, Rizal)

34. Dr. Jovito Cariño (University of Santo Tomas, Manila) 
35. Mr. Bernardo Caslib, Jr. (Ateneo de Manila University, Quezon City)

36. Mr. Delfin P. Chiniona (Technological Institute of the Philippines, Manila)

37. Dr. Dalmacito A. Cordero Jr. (De La Salle University, Manila)

38. Dr. Franz Guiseppe Cortez (University of Santo Tomas, Manila)

39. Mr. Roy John R. Del Rosario (Holy Angel University, Angeles City, Pampanga)

40. Ms. Amanda Juico Dela Cruz (University of the Philippines - Diliman)

41. Fr. Prudencio M.Edralin Ph.D. (Thomas Aquinas Major Seminary/Lyceum of Aparri)

42. Fr. Francis Paul Escaño, RCJ (Rogationist Seminary College, Cebu City)

43. Mr. Joven B. Esteban (Holy Angel University, Angeles City, Pampanga)

44. Dr. Edwin Etiyiebo (University of the Witwatersrand, Johannesburg, South Africa)

45. Mr. Ismael Fortunado (Filipino Inventors Society, Inc.)

46. Fr. Lemeul W. Galias, SMAHE (Holy Family Center of Studies Foundation, Sorsogon)

47. Mr. Prince Airick S. Gapo (Fiat Lux Academe-Cavite)

48. Mr. Aris S. Gonzales (Holy Angel University, Angeles City, Pampanga)

49. Dr. Eden M. Gripaldo (University of the Philippines - Diliman, Quezon City)

50. Dr. Ria Edwina M. Gripaldo (Mayo Clinic Rochester, Minnesota, USA)

51. Mr. Michael Roland F. Hernandez (Ateneo de Naga University, Naga City)

52. Ms. Ma. Rubeth Hipolito (Holy Angel University, Angeles City, Pampanga)

53. Dr. Mark Omorovie Ikeke (Delta State University, Abraka, Nigeria)

54. Fr. Damian Ilodigwe ( Ss. Peter and Paul Seminary, Ibadan, Nigeria)

55. Mr. Aldrin Salvador Jaramilla (University of Northern Philippines, Vigan City)

56. Mr. Joseph Martin M. Jose (De La Salle University - Manila)

57. Dr. Miguel López-Astorga (Talca University, Chile)

58. Mr. Welfredo Q. Mamaril (Holy Angel University, Angeles City, Pampanga)

59. Mr. Gil Lopez Manahan (University of the East-Manila)

60. Mr. Edmund M. Maniago (Holy Angel University, Angeles City, Pampanga)

61. Mr. Angelito L. Martinez (Holy Angel University, Angeles City, Pampanga)

62. Ms. Louziela P. Masana (Cavite State University-General Trias City Campus, Cavite)

63. Mr. Francis T. Matay-eo, Jr. (Isabela State University, Isabela)

64. Dr. Rhochie Avelino Matienzo (University of Santo Tomas, Manila)

65. Dr. Robert Montaña (University of Santo Tomas, Manila)

66. Dr. Nguyen Thi Minh Ngoc (Institute of Sociology, Ha Noi, Vietnam)

67. Dr. Charles C. Nweke (Nnamdi Azikiwe University, Awka, Nigeria)

68. Dr. Ian Raymond Pacquing (University of Santo Tomas, Manila)

69. Mr. Erwin C. Pagtalunan (Marcelo H. Del Pilar National High School, Malolos)

70. Mr. Joseph Pana (De La Salle University, Manila)

71. Dr. Engelbert C. Pasag (PanPacific University, Urdaneta City)

72. Mr. Beaujorne Sirad Ramirez (Immaculate Conception Academy_Greenhills, San Juan)

73. Mr. Mickael A. Raymundo (Bambang National High School, Nueva Vizcaya)

74. Mr. Adrian V. Remodo (Ateneo de Naga University, Naga)

75. Mr. Marc Rambo D. Rondera (National University-Manila)

76. Mr. David Floyd M. Roxas (Bulacan State University, Malolos, Bulacan)

77. Dr. Alfredo B. Santos ((Holy Angel University, Angeles City, Pampanga)

78. Mr. Virgilio A. Rivas (Polytechnic University of the Philippines, Manila) 